\title{
Propagation Characteristics of Human Body Communication in Brain-Controlled Functional Electrical Stimulation
}

\author{
Jingzhen Li, Yuhang Liu, Zedong Nie \\ Shenzhen Institutes of Advanced Technology, Chinese Academy of Science \\ Shenzhen 518055, China \\ zd.nie@siat.ac.cn
}

\begin{abstract}
Human body communication (HBC), which uses the human body as a propagation medium, is a promising communication technology in brain-controlled functional electrical stimulation (FES). In order to study the propagation characteristics of HBC channel between intra-brain and body implant, a 45kg pig was employed as an experimental subject in this paper. The channel characteristics of amplitude and group delay were investigated by vector network analyzer (VNA) over different frequencies (from $0.3 \mathrm{MHz}$ to $200 \mathrm{MHz}$ ). A battery powered transmitter and receiver were adopted to study the influence of electrode size and type. Experimental results show that the optimal HBC frequency for brain-controlled FES applications is from $5 \mathrm{MHz}$ to $50 \mathrm{MHz}$. Electrode size have little impact on signal transmission in body channel. In addition, signal transmission is insensitive with the electrode type.
\end{abstract}

\section{Categories and Subject Descriptors} B.4.2 [INPUT/OUTPUT AND DATA COMMUNICATIONS]: Input / Output Devices -Channels and controllers

\section{C.2.1 [COMPUTER-COMMUNICATION NETWORKS]: \\ Network Architecture and Design-Wireless communication}

\section{General Terms}

Measurement, Performance, Design, Experimentation, Human Factors, Verification

\section{Keywords}

Human body communication, Propagation characteristics, Channel, Electrode, Brain-controlled functional electrical stimulation

\section{INTRODUCTION}

It is reported that more than 130,000 people each year suffer from spinal cord injury all over the world [1]. Spinal cord injury leads to the paralysis of limbs, which has a great impact on daily activities. For paralyzed patients, a traditional rehabilitation method is functional electrical stimulation (FES), which attempts to restore motor behaviors to paralyzed limbs by electrically stimulating nerves or muscles [2, 3]. However, FES is restricted to several grasp patterns by using pre-programmed stimulus. Moreover, the stimulus is not controlled by human brain. Recently, brain-controlled FES, which adopts the brain-neural signals to control electrical stimulus, is regarded as a potential treatment to restore the goal-directed movement of paralyzed limbs [4]. Several primary investigations of brain-controlled FES were conducted in $[5,6]$. Neural signals for movement were firstly acquired from the electrodes that were implanted in intrabrain. Then the neural signals were converted to stimulator commands, and then the stimulator commands were transmitted to stimulator, which was implanted in the forearm of experimental subject. At last, stimulator generated electrical stimulus to restore the movement of paralyzed arm. In aforementioned works, a robust, low power and security communication between intrabrain and body implant plays an important role in brain-controlled FES. The traditional wire communication and wireless communication technologies would be considered in braincontrolled FES. However, wire communication is inconvenient to wear. Moreover, wire communication involves a high risk of infection. Wireless communications, such as Bluetooth, Zigbee and other commercial wireless communications, have the risk of inter-network interference and unauthorized interception of information due to the communication distance is up to dozens of meters [7]. In addition, the high power consumption and low data rate are also the limitations when the traditional wireless communications are applied in brain-controlled FES [8].

Human body communication (HBC), which uses the human body as a propagation medium, is a prospective solution for body area networks [9, 10]. Compared with other traditional wireless communications, HBC has the advantage of low power consumption, high efficiency and high data rate $[11,12]$. Furthermore, HBC is motion insensitive and environmental independent [13]. Therefore, HBC is regarded as a promising communication in brain-controlled FES. However, thus far, few papers have tackled the propagation characteristics of HBC channel between intra-brain and body implant.

In this paper, $\mathrm{HBC}$ is proposed as a communication technology for brain-controlled FES. We investigated the propagation characteristics of the channel between intra-brain and body implant through VNA. The influence of electrode size and electrode type to signal transmission in $45 \mathrm{MHz}$ had also been demonstrated by a battery powered transmitter and receiver. This 
paper is structured into four sections. Section 2 is the experimental setup about HBC in a Bama pig. Section 3 explains the measurement results. The final section is conclusion.

\section{EXPERIMENTAL SETUP}

In order to analyze the propagation characteristics of HBC channels, experimental measurement is conducted. A liquid phantom is difficult to represent the electrical properties of human's tissue because the liquid phantom is a homogeneous medium. Considering the electrical properties of mammal's tissue and geometric shape are similar to human, a Bama pig, weighting $45 \mathrm{~kg}$, was used as an experimental subject in our investigation. All surgical and experimental procedures have been approved by the Institutional Animal Care and Use Committee (IACUC, No.S20150125-01). The experimental equipments included VNA, transmitter, receiver and electrodes.

\subsection{Vector Network Analyzer}

Vector network analyzer (VNA, Agilent E5061A) is used to analyze the amplitude and group delay of different channels. The frequency sweep range is set from $0.3 \mathrm{MHz}$ to $200 \mathrm{MHz}$. The transmitting electrode and receiving electrode are connected to the Port 1 and Port 2 of VNA, respectively.

\subsection{Transmitter and Receiver}

A battery powered transmitter and receiver, which are designed by our team, are adopted to investigate the influence of electrode size and type to signal transmission in particular channel and frequency. The transmitter incorporates a direct digital synthesizer and a microprocessor. The transmitter is able to output a sine wave at $45 \mathrm{MHz}$. The output power of sine wave is $0 \mathrm{dBm}$. The receiver consists of a $45 \mathrm{MHz}$ band pass crystal filter, a logarithmic power detector and a microprocessor. There is a 10bit analog-to-digital converter integrates in the microprocessor. The measurement data are analog-to-digital converted and stored to secure digital card for off-line analysis. Fig.1 illustrates the calibrated relationship between the input power $(\mathrm{dBm})$ and digital output (10-bit A/D converter).

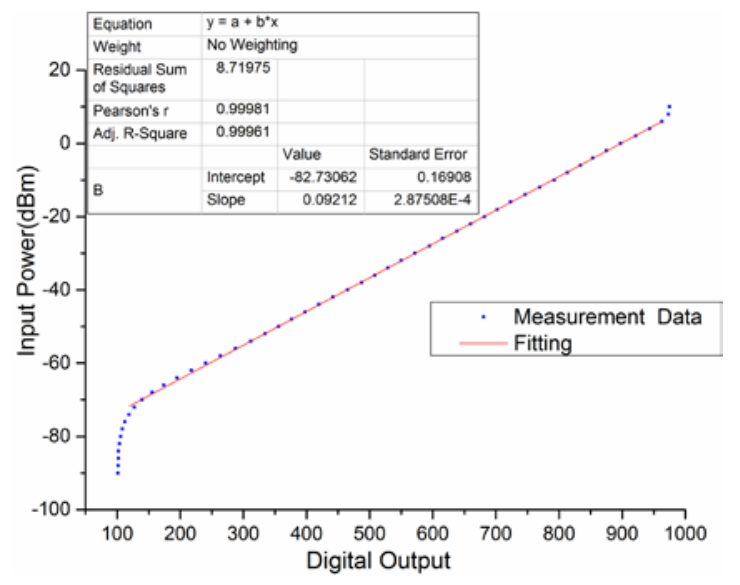

Figure 1. Calibrated relationship between the input power and digital output from 10-bit $\mathrm{A} / \mathrm{D}$ converter.

\subsection{Electrode}

Electrodes are adopted to achieve signal transmitting and receiving. Two types of electrodes are utilized in this paper. The conducting electrode is made of copper and coaxial cable. The insulated electrode, whose the surface is insulated, is made of copper, coaxial cable and polyvinyl chloride (PVC) tape. Two sizes of conducting electrodes are also used in the experiment. Fig.2 demonstrates the pictures of electrode.

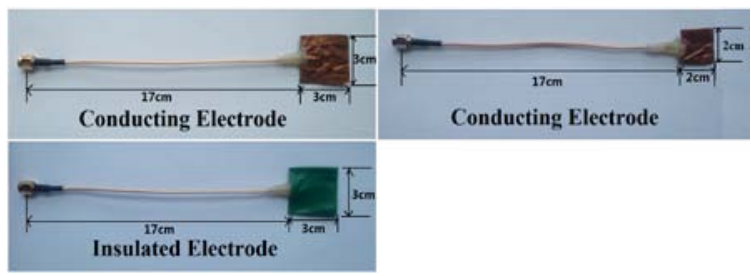

Figure 2. The pictures of electrode.

\subsection{Experiment Setup}

At the beginning of experiment, a pig was practiced euthanasia via anesthetic injection. Then an electrode was implanted in the pig's intra-brain by surgical persons after the skull was opened. Some positions of pig's body were gashed through T cut method. The incisions were sutured after the electrodes were implanted in the incisions. Three experiments were set up in the paper. All experiments were performed in an animal anatomy laboratory. The pig was placed on the test-bed and the electrodes were implanted in pig's intra-brain and body.

As Fig.3 showed, in order to investigate the propagation characteristics of different channels, four channels were measured by VNA in experiment I . Specifically, the transmitting electrode was implanted in pig's intra-brain. The receiving electrodes were implant in pig's back, belly, left foreleg and left hind leg, respectively. The amplitude and group delay of different channels were considered in our investigation. Snapshots of data $(1,601)$ were acquired from VNA in every experiment when the frequency sweep range was set from $0.3 \mathrm{MHz}$ to $200 \mathrm{MHz}$.

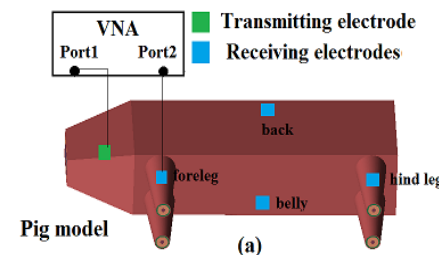

(a)

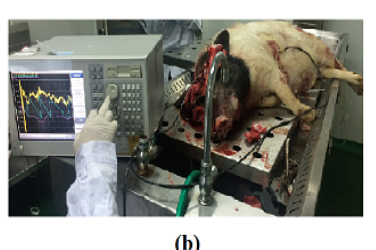

(b)
Figure 3. (a)Diagram of experiment I ; (b)Measurement of amplitude and group delay via VNA.

In experiment $\mathbb{I}$, in order to analyze the influence of electrode size to signal transmission, the $2 \mathrm{~cm} \times 2 \mathrm{~cm}$ and $3 \mathrm{~cm} \times 3 \mathrm{~cm}$ electrodes were utilized. Fig. 4 is the diagram of experimental setup and experimental process. The power variation of the channel between the pig's intra-brain and left foreleg was investigated when the transmitter (TX) and receiver (RX) were connected different sizes of electrodes. Four comparative experiments were conducted and 2,000 valid data were collected in each experiment.

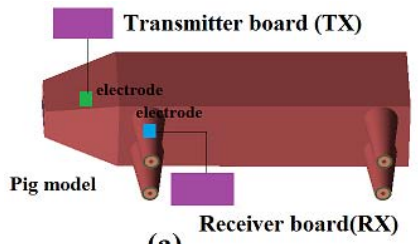

(a)

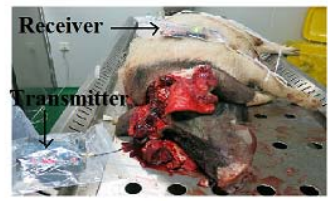

(b)
Figure 4. (a) Diagram of experiment II ; (b) Measurement of different electrode size via transmitter and receiver. 
In experiment $\mathrm{II}$, the influence of electrode type to signal transmission was considered via the battery powered transmitter and receiver. The conducting electrode and insulated electrode were adopted in this experiment. Transmitter (TX) and receiver (RX) were connected different types of electrodes. The channel is from pig's intra-brain to belly. The process of experiment III is similar to experiment II. Four comparative experiments were executed and 2,000 valid data were collected in each experiment.

The specifications of all the experiments that we have been conducted are as the table I listed.

Table 1. Experiments description

\begin{tabular}{|c|c|c|c|}
\hline & $\begin{array}{l}\text { Electrode } \\
\text { Size }\end{array}$ & $\begin{array}{l}\text { Electrode } \\
\text { Type }\end{array}$ & $\begin{array}{c}\text { Experimental } \\
\text { Description }\end{array}$ \\
\hline $\begin{array}{l}\text { EXP } \\
. \mathrm{I}\end{array}$ & $\begin{array}{l}\text { TX:3cm } \times \\
3 \mathrm{~cm} \\
\text { RX:3cm× } \\
3 \mathrm{~cm}\end{array}$ & $\begin{array}{c}\text { TX: conducting } \\
\text { electrode } \\
\text { RX: conducting } \\
\text { electrode }\end{array}$ & $\begin{array}{c}\text { Research propagation } \\
\text { characteristics of four } \\
\text { channels via the VNA } \\
\text { from } 0.3 \mathrm{MHz} \text { to } \\
200 \mathrm{MHz}\end{array}$ \\
\hline $\begin{array}{l}\text { EXP } \\
\text {. II }\end{array}$ & $\begin{array}{l}\text { TX: } \\
\text { various } \\
\text { RX: } \\
\text { various }\end{array}$ & $\begin{array}{c}\text { TX: conducting } \\
\text { electrode } \\
\text { RX: conducting } \\
\text { electrode }\end{array}$ & $\begin{array}{c}\text { Research the } \\
\text { influence of electrode } \\
\text { size via transmitter } \\
\text { and receiver at } 45 \\
\text { MHz }\end{array}$ \\
\hline $\begin{array}{l}\text { EXP } \\
\text {. III }\end{array}$ & $\begin{array}{l}\mathrm{TX}: 3 \mathrm{~cm} \times \\
3 \mathrm{~cm} \\
\mathrm{RX}: 3 \mathrm{~cm} \times \\
3 \mathrm{~cm}\end{array}$ & $\begin{array}{lc}\text { TX: } & \text { various } \\
\mathrm{RX}: & \text { various }\end{array}$ & $\begin{array}{c}\text { Research the } \\
\text { influence of electrode } \\
\text { type via transmitter } \\
\text { and receiver at } 45 \\
\text { MHz }\end{array}$ \\
\hline
\end{tabular}

\section{RESULTS AND DISCUSSION}

\subsection{Propagation Characteristic of Different Channels}

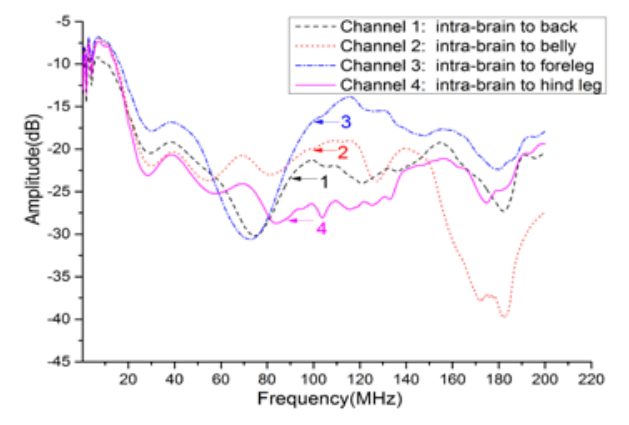

Figure 5. The characteristics of amplitude in four channels.

The amplitude of channel plays an important role in wireless communication. It determines the transmitter power, receiver sensitivity and other parameters. It is favorable to reduce the complexity of transceiver if the attenuation of amplitude is small and not related to channels. Fig.5 illustrates the propagation characteristics of amplitude (in $\mathrm{dB}$ ) in different channels, while the frequency sweep range of VNA is from $0.3 \mathrm{MHz}$ to $200 \mathrm{MHz}$. The amplitude of all channels is over $-8 \mathrm{~dB}$ when frequency is $10 \mathrm{MHz}$. The channels have a low pass characteristic. With the increase of frequency, the amplitude of channel 1 is fading. Other channels also have a similar fading. Meanwhile, all four channels have a peak value of amplitude at $40 \mathrm{MHz}$. In addition, the trend of amplitude is independent of body channels when frequency is below $50 \mathrm{MHz}$. However, the fluctuations of amplitude in four channels are different when the frequency is over $50 \mathrm{MHz}$. The amplitude of channel 1 and channel 3 is the smallest when the frequency is about $70 \mathrm{MHz}$. For channel 2 and channel 4, the minimum value of amplitude is $-40 \mathrm{~dB}$ at $180 \mathrm{MHz}$ and $-28 \mathrm{~dB}$ at $85 \mathrm{MHz}$, respectively. Therefore, the channel has a great impact on signal amplitude if the frequency is over $50 \mathrm{MHz}$.

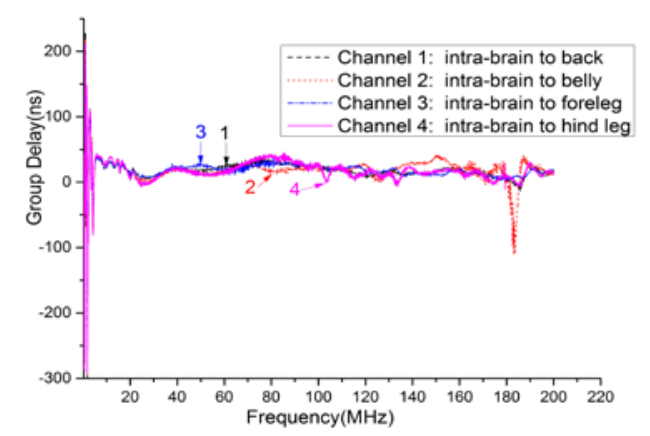

Figure 6. The characteristics of group delay in four channels.

Group delay reflects the delay time between output envelopes and input envelopes. It is an index to evaluate the phase nonlinearity and wave distortion. The transmittal signal has least distortion if group delay is constant. Fig.6 demonstrates the propagation characteristics of group delay (in ns) in different channels. The curves of group delay in all four channels are similar when the frequency sweep range is from $0.3 \mathrm{MHz}$ to $180 \mathrm{MHz}$. Thus, the group delay is almost independent of body channels. The fluctuation range of each channel is approximately from -300ns to 200ns when frequency is below $0.5 \mathrm{MHz}$. With the increase of frequency, the fluctuations of group delay in all four channels have weakened. The fluctuation range of channel 1 is between $-5 \mathrm{~ns}$ and $38 \mathrm{~ns}$ when the frequency is from $5 \mathrm{MHz}$ to $180 \mathrm{MHz}$. Other channels also have the similar fluctuation range. Therefore, the curves of group delay at all four channels are relatively smooth. In other word, the group delay of channels is more stable when the frequency is from $5 \mathrm{MHz}$ to $180 \mathrm{MHz}$.

According to the measurements of amplitude and group delay, the frequency from $5 \mathrm{MHz}$ to $50 \mathrm{MHz}$ is supposed to be the optimal frequency in HBC channels. The trend of amplitude is independent of channels. Meanwhile, the group delay of channels is stable.

\subsection{Influence of Electrode Size}

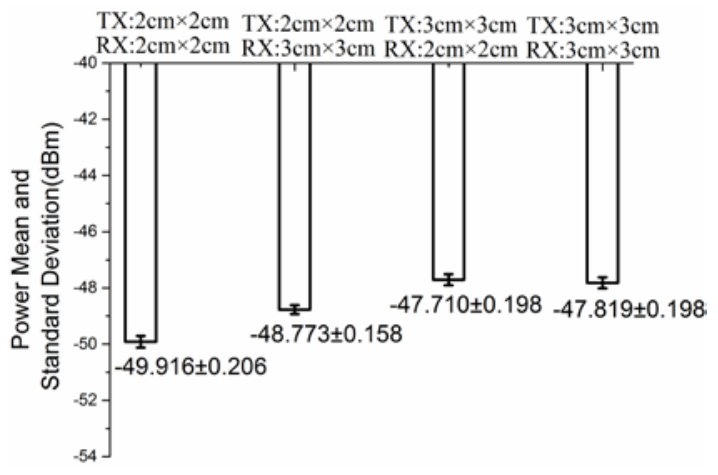

Figure 7. The influence of electrode size. 
Fig.7 describes the influence of electrode size to signal transmission. Typically, the received signal power are -45.063 $\mathrm{dBm},-46.425 \mathrm{dBm},-47.710 \mathrm{dBm}$ and $-46.336 \mathrm{dBm}$, respectively, when the transmitter (TX) and receiver (RX) are connected different sizes of electrodes. Furthermore, the power standard deviations are $0.206 \mathrm{~dB}, 0.158 \mathrm{~dB}, 0.198 \mathrm{~dB}$ and $0.198 \mathrm{~dB}$ in all four comparative experiments. Experimental results show that electrode size has little impact on signal transmission. The electrode size plays an important role in the miniaturization of HBC system. Therefore, a promising method to achieve miniaturization is adopting small size electrode due to the independence of electrode size to signal transmission.

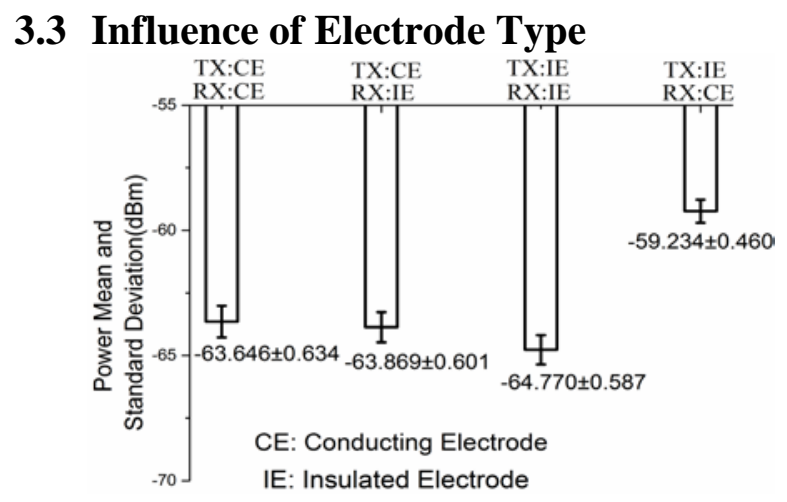

Figure 8. The influence of electrode type.

Fig.8 illustrates the influence of electrode type to signal transmission. The received signal power is $-59.234 \mathrm{dBm}$ when transmitter (TX) is connected insulated electrode and receiver (RX) is connected conducting electrode in the fourth experiment. In addition, the received signal power of other experiments is approximately $-64 \mathrm{dBm}$. Furthermore, the power standard deviation is similar in all four comparative experiments. Therefore, overall, signal transmission is insensitive with electrode type. From the point of biological compatibility, the material of electrode is flexible because the electrode type has little impact on signal transmission.

\section{CONCLUSION}

In this paper, the propagation characteristics of channel between pig's intra-brain and body implant were investigated. The frequency from $5 \mathrm{MHz}$ to $50 \mathrm{MHz}$ is supposed to be the optimal frequency in HBC channels. The trend of amplitude is independent of channels when frequency is from $5 \mathrm{MHz}$ to $50 \mathrm{MHz}$. Meanwhile, the group delay of channels is stable. Experimental results show that electrode size and electrode type have little impact on signal transmission. The independent of electrode size is helpful to achieve the miniaturization of HBC system. The insensitivity of electrode type indicates that the choice of electrode material in $\mathrm{HBC}$ is flexible. Overall, from the preliminary results, HBC is appropriate for the communication in brain-controlled FES. In the next phase, we will demonstrate statistical significance of HBC channels through a large of experiments.

\section{ACKNOWLEDGMENTS}

This study was financed partially National Natural Science Funds of China (Grant No.61403366), Shenzhen Basic Research Project
Fund (JCYJ20140417113430695), Shenzhen Technology Development Project Fund (CXZZ20150505093829778).

\section{REFERENCES}

[1] International. Campaign for Cures of Spinal Cord Injury Paralysis. http://www.campaignforcure.org

[2] A. Farhoud and A. Erfanian. 2014. Fully Automatic Control of Paraplegic FES Pedaling Using Higher-Order Sliding Mode and Fuzzy Logic Control. Neural Systems and Rehabilitation Engineering, IEEE Transactions on, vol. 22, (Jan. 2014) ,533-542.

[3] C. L. Lynch and M. R. Popovic. 2008. Functional Electrical Stimulation. Control Systems, IEEE, vol. 28, (2008), 40-50.

[4] C. T. Moritz, S. I. Perlmutter, and E. E. Fetz. 2008. Direct control of paralysed muscles by cortical neurons. Nature, vol. 456, (Dec. 2008), 639-42.

[5] Eric A. Pohlmeyer, Emily R. Oby, and Eric J. Perreault. 2009. Toward the Restoration of Hand Use to a Paralyzed Monkey: Brain-Controlled Functional Electrical Stimulation of Forearm Muscles. PloS one, vol. 4, (June 2009), 1-8.

[6] C. Ethier, E. R. Oby, M. J. Bauman, and L. E. Miller. 2012. Restoration of grasp following paralysis through braincontrolled stimulation of muscles. Nature, vol. 485, (May 2012), 368-371.

[7] L. Jingli, X. Liu, and A. Swami. 2009. Collision analysis for coexistence of multiple bluetooth piconets and WLAN with dual channel transmission. Communications, IEEE Transactions on, vol. 57, (Apr. 2009), 1129-1139.

[8] M. Patel and W. Jianfeng. 2010. Applications, challenges, and prospective in emerging body area networking technologies. Wireless Communications, IEEE, vol. 17, (2010), 80-88.

[9] T. G. Zimmerman.1996. Personal Area Networks: Near-field intrabody communication. IBM Systems Journal, vol. 35, (1996), 609-617.

[10] T. N. Xuan, D. Muramatsu, and K. Sasaki. 2015. Measurement of human body communication transmission characteristics at $20 \mathrm{MHz}$. in Medical Information and Communication Technology (ISMICT), 2015 9th International Symposium on, (Mar. 2015), 45-48.

[11] B. Joonsung, S. Kiseok, and L. Hyungwoo.2012. A 0.24$\mathrm{nJ} / \mathrm{b}$ Wireless Body-Area-Network Transceiver with Scalable Double-FSK Modulation. Solid-State Circuits, IEEE Journal of, vol. 47, (Nov, 2012), 310-322.

[12] Fazzi A., Ouzounov S., van den Homberg. J. 2009. A $2.75 \mathrm{~mW}$ wideband correlation-based transceiver for bodycoupled communication. proceedings of the Solid-State Circuits Conference - Digest of Technical Papers, 2009 ISSCC 2009 IEEE International, (Feb. 2009),8-12.

[13] Z. Nie, J. Ma, Z. Li, H. Chen, and L. Wang. 2012. Dynamic propagation channel characterization and modeling for human body communication. Sensors (Basel), vol. 12, (Dec. 2012), 17569-17587. 